Article

\title{
Plant-Pathogen Interaction, Circadian Rhythm, and Hormone-Related Gene Expression Provide Indicators of Phytoplasma Infection in Paulownia fortunei
}

\author{
Guoqiang Fan *, Yanpeng Dong, Minjie Deng, Zhenli Zhao, Suyan Niu and Enkai Xu \\ Institute of Paulownia, Henan Agricultural University, 95 Wenhua Road, Jinshui District, \\ Zhengzhou 450002, China; E-Mails: dongdyp@163.com (Y.D.); dengmj1980@126.com (M.D.); \\ zhaozh12006@126.com (Z.Z.); suyanniu_happy@126.com (S.N.); xuenkai19831206@163.com (E.X.) \\ * Author to whom correspondence should be addressed; E-Mail: fanguoqiangdr@163.com; \\ Tel./Fax: +86-371-6355-8605.
}

External Editor: Chang Won Choi

Received: 16 October 2014; in revised form: 20 November 2014 / Accepted: 2 December 2014 / Published: 12 December 2014

\begin{abstract}
Phytoplasmas are mycoplasma-like pathogens of witches' broom disease, and are responsible for serious yield losses of Paulownia trees worldwide. The molecular mechanisms of disease development in Paulownia are of considerable interest, but still poorly understood. Here, we have applied transcriptome sequencing technology and a de novo assembly approach to analyze gene expression profiles in Paulownia fortunei infected by phytoplasmas. Our previous researches suggested that methyl methane sulfonated (MMS) could reverse the effects of the infection. In this study, leaf samples from healthy, infected, and both infected and methyl methane sulfonate treated plants were analyzed. The results showed that the gene expression profile of P. fortunei underwent dramatic changes after Paulownia witches' broom (PaWB) phytoplasma infection. Genes that encoded key enzymes in plant-pathogen interaction processes were significantly up-regulated in the PaWB-infected Paulownia. Genes involved in circadian rhythm and hormone-related genes were also altered in Paulownia after PaWB infection. However, after the PaWB-infected plants were treated with MMS, the expression profiles of these genes returned to the levels in the healthy controls. The data will help identify potential PaWB disease-resistance genes that could be targeted to inhibit the growth and reproduction of the pathogen and to increase plant resistance.
\end{abstract}


Keywords: Paulownia fortunei; Paulownia witches' broom (PaWB); phtoplasma; de novo assembled transcriptome; disease resistance gene

\section{Introduction}

Paulownia witches' broom (PaWB) disease is a devastating disease of Paulownia trees, which can result in significant economic losses over a large part of the world [1,2]. Infected plants develop a series of similar symptoms, such as witches' brooms, short internodes, yellowing or reddening of leaves, phyllody, stunting and decline, virescence, sterile flowers and necrosis, and altered volatile production [3]. It has been reported that the disease is caused by an obligate biotrophic plant pathogen called phytoplasma, which belongs to the taxon "Candidatus Phytoplasma australiense" International Research Programme on Comparative Mycoplasmology (IRPCM), Phytoplasma Taxonomy Group 2004 [4]. Phytoplasma are mycoplasma-like organisms that reside in the phloem sieve cells of a plant and are transmitted by phloem-sucking insects [5]. They belong to the Bacteria Kingdom. After residence, they can travel systemically through the pores of the sieve plates and appear to accumulate mainly in roots, developing leaves, and flowers. Phytoplasmas have no cell wall and are strictly biotrophic; thus, they can survive only on living host cells and are quite difficult to study.

Studies of PaWB disease during the last 30 years have been focused mainly on the ecological and biological characteristics of the disease, while the mechanisms behind it are largely unknown. Although researchers recognize that understanding the mechanisms is important, studying them is difficult largely because phytoplasma have never been cultured [6]. Recently, our studies showed that the infected Paulownia seedlings could recover a healthy morphology by treatment with suitable concentration of methyl methane sulfonate (MMS), in which the phytoplasma could be removed [7-9].

Some molecular biology-based methods have been developed to detect the existence of phytoplasma [10,11] and more information about PaWB disease has been explored. However, a comprehensive view of the process has yet to be formed. Recently, next-generation sequencing has been used to study this complicated biological system. Mou et al. [12] conducted a transcriptome study on healthy and phytoplasma-infected Paulownia tomentosa $\times$ Paulownia fortunei samples using RNA-Seq. At about the same time, our team [13] reported a similar study. There are two main differences between these two studies: (1) the samples used in our research came from tissue cultured plants, while the samples used by Mou et al. were both tissue cultured plants and field grown ones. However, in our study, samples that had been treated with MMS were included; (2) the results of the two studies differ from each other. Different genes were found differentially expressed in the infected Paulownia. We proposed a hypothesis to interpret the possible functional roles of the identified differentially expressed genesin Paulownia-phytoplasma compatible interactions, while Mou et al. found that genes encoding key enzymes in cytokinin biosynthesis were significantly induced in the phytoplasma-infected samples, and genes involved in cell wall biosynthesis and degradation were largely up-regulated while genes related to photosynthesis were down-regulated in the phytoplasma-infected samples.

In the present research, we studied the effects of MMS on the morphological changes of $P$. fortunei seedlings with PaWB disease and found that the seedlings treated with $60 \mathrm{mg} \cdot \mathrm{L}^{-1}$ MMS for more than 
$3 \mathrm{~h}$ showed no disease symptoms. After that, we performed a transcriptomic analysis using the Illumina/SolexaGAIIx platform and de novo assembly to investigate the differences among three P. fortunei samples: PF (healthy), PFI (phytoplasma-infected) and PFI-60 (phytoplasma-infected and $60 \mathrm{mg} \cdot \mathrm{L}^{-1}$ MMS-treated). Our study demonstrated that dramatic changes in gene expression occurred in the phytoplasma infection process, while after MMS treatment, the expression profile of a large number of genes changed back to the profile of the healthy sample. Our study has provided genome-wide potential phytoplasma-resistant genes and inherent pathways that are candidates for further study. The results presented provide a comprehensive platform on which our understanding of the complicated biological process of plant-phytoplasma interactions can be built.

\section{Results}

\subsection{Detection of Paulownia Witches' Broom (PaWB) Phytoplasma in Paulownia Showing} Disease Symptoms

After PaWBphytoplasma infection, the Paulownia plants showed mainly typical disease symptoms, including witches' broom, yellowing of leaves, smaller leaves, short internodes, phyllody, and sterile flowers. When the concentration of the MMS was less than $20 \mathrm{mg} \cdot \mathrm{L}^{-1}$, the PaWB seedlings retained an unhealthy morphology. After $20-60 \mathrm{mg} \cdot \mathrm{L}^{-1}$ MMS treatment, the PaWB seedlings regained a healthy morphology. When the concentration of MMS was above $60 \mathrm{mg} \cdot \mathrm{L}^{-1}$, the growth of the PaWB seedlings was inhibited (Figure 1). The 16SrDNA of PaWB phytoplasma is $1.24 \mathrm{~kb}$ long [14]. During our study, fragments of the 16SrDNA were detected in the midribs of the PFI and PFI-20 (phytoplasma-infected and $20 \mathrm{mg} \cdot \mathrm{L}^{-1}$ MMS-treated) samples, but not in the PF and PFI-60 samples (Figure 2). These results indicated that the PaWB infected seedlings recovered a healthy morphology after treatment with an optional concentration of MMS.

Figure 1. Methyl methane sulfonate (MMS) treated plantlets morphology. (A) Paulownia witches' broom (PaWB)-infected plantlets, symptoms such as witches' brooms were shown; (B) $20 \mathrm{mg} \cdot \mathrm{L}^{-1} \mathrm{MMS}$; (C) $60 \mathrm{mg} \cdot \mathrm{L}^{-1} \mathrm{MMS}$; (D) $100 \mathrm{mg} \cdot \mathrm{L}^{-1} \mathrm{MMS}$; (E) Healthy plantlets.

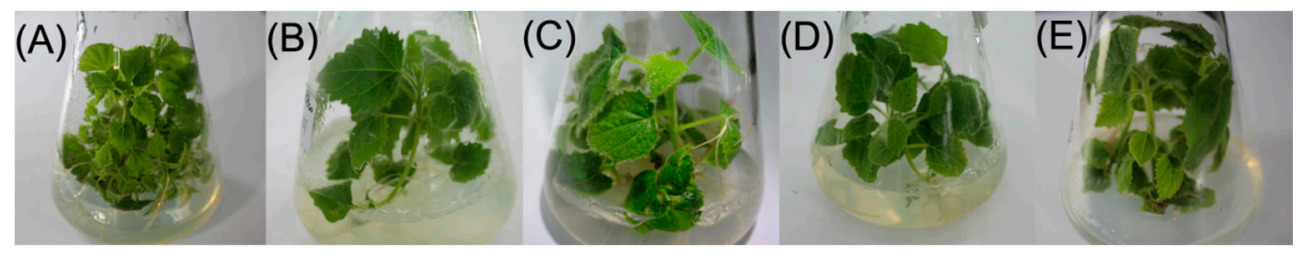

Figure 2. Phytoplasma 16SrDNA amplication in MMS treated plantlets. (1) PaWB-infected plantlets; (2) $20 \mathrm{mg} \cdot \mathrm{L}^{-1} \mathrm{MMS}$; (3) $60 \mathrm{mg} \cdot \mathrm{L}^{-1} \mathrm{MMS}$; (4) $100 \mathrm{mg} \cdot \mathrm{L}^{-1} \mathrm{MMS}$; (5) Healthy plantlets; (6) ddH $\mathrm{H}_{2} \mathrm{O}$; (M) DNA Marker.

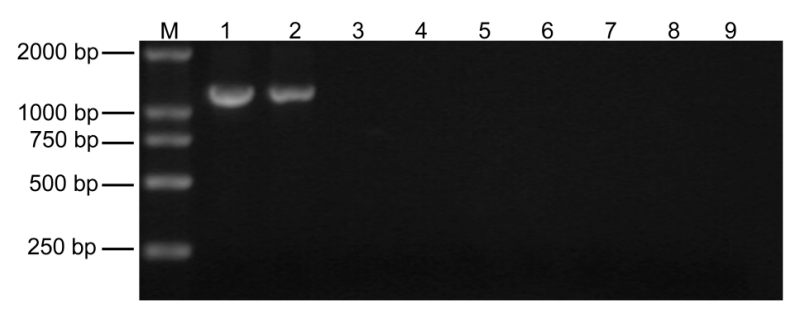




\subsection{Transcriptome Sequencing and Assembly}

The Illumina Solexa sequencing generated 57,846,728 high-quality paired-end reads, 58,451,828 high-quality paired-end reads, and 51,417,240 high-quality paired-end reads for the PF, PFI, and PTFI-60 libraries, respectively. The lengths of the reads were between 90 and $100 \mathrm{bp}$. All the high-quality reads in the three libraries were assembled into contigs, which were further assembled into unigenes. From the PF, PFI, and PFI-60 libraries, the assembly produced 116,749, 91,997, and 103,300 unigenes with average lengths of 1016, 864 and 1029 bp, and median lengths (N50) of 1963, 1715, and 1899 bp, respectively (Table 1). Together we obtained a total of 115,116 non-redundant unigenes (all unigenes) with a mean length of $1282 \mathrm{bp}$, and N50 of $2067 \mathrm{bp}$. Among these unigenes, 75,498 (65.58\%) were longer than $500 \mathrm{bp}$, and 55,029 (47.80\%) were longer than 1000 bp (Figure S1).

Table 1. Overview of the sequencing and assembly of the transcriptome of $P$. fortunei.

\begin{tabular}{llll}
\hline Statistics of Data Production & PF & PFI & PFI-60 \\
\hline Number of high-quality reads & $57,846,728$ & $58,451,828$ & $51,417,240$ \\
GC percentage (\%) & $46.30 \%$ & $46.39 \%$ & $46.39 \%$ \\
Contigs & PF & PFI & PFI-60 \\
Number of contigs & 174,105 & 155,253 & 175,247 \\
Average length of contigs (nt) & 358 & 361 & 339 \\
Length of N50 (bp) & 670 & 740 & 654 \\
Unigenes & PF & PFI & PFI-60 \\
Number of unigenes & 116,749 & 91,997 & 103,300 \\
Length of N50 (bp) & 1963 & 1715 & 1899 \\
Average length of unigenes (bp) & 1016 & 864 & 1029 \\
\hline All unigenes & & & \\
Number of all unigenes & 115,116 & & \\
Length of N50 (bp) & 2067 & & \\
Average length of all unigenes (bp) & 1282 & & \\
\hline
\end{tabular}

\subsection{Annotation of Non-Redundant Unigenes}

Because the available genomic information for Paulownia is limited, a BLASTX search with a threshold of $1.0 \times 10^{-5}$ was performed to predict the functions of the all unigenes against six public databases (nr, nt, Swiss-Prot, KEGG (Kyoto Encyclopedia of Genes and Genomes), COG (Cluster of Orthologous Groups of proteins), and GO (Gene Ontology)). We found that 79,035 unigenes (68.66\% of all unigenes) shared similarities with known sequences (Table 2).

Among the unigenes that matched known sequences, $74.3 \%$ showed strong homology $\left(E\right.$-value $\left.<1.0 \times 10^{-30}\right)$, and $25.7 \%$ showed some homology $\left(1.0 \times 10^{-30}<E\right.$-value $\left.<1.0 \times 10^{-5}\right)$ with $\mathrm{nr}$ sequences (Figure $3 \mathrm{~A}$ ). The similarity distribution showed that $22.1 \%$ of the unigenes had similarities higher than $80 \%$, and $77.9 \%$ had similarities of $17 \%-80 \%$ (Figure 3B). The species distribution showed that the unigenes had best matches to sequences from Vitis vinifera (51.9\%), followed by Ricinus communis (13.0\%), Populus trichocarpa (10.8\%), and Glycine max (5.6\%) (Figure 3C). Of the 115,116 all unigenes, $32,269(28.0 \%)$ were assigned to 25 COG categories (Figure 4), 63,555 (55.2\%) were assigned to at least one GO term (Figure 5), and 46,381 (40.3\%) were mapped to 128 KEGG pathways (Table S1). 
Table 2. Annotation of non-redundant unigenes of the transcriptome of $P$. fortunei.

\begin{tabular}{ccc}
\hline Database & Number of Annotated Unigenes & Percentage of Annotated Unigenes (\%) \\
\hline $\mathrm{nr}$ & 76,507 & 66.5 \\
$\mathrm{nt}$ & 69,981 & 60.8 \\
Swiss-Prot & 49,385 & 42.9 \\
KEGG & 46,381 & 40.3 \\
COG & 32,269 & 28.0 \\
GO & 63,555 & 55.2 \\
All & 79,035 & 68.7 \\
\hline
\end{tabular}

Figure 3. Distribution of $E$-values (A); similarity (B); and species (C) for the allunigene BLAST (Basic Local Alignment Search Tool) matches against the nr-protein database. For the BLASTX (Basic Local Alignment Search Tool, search protein database using a translated nucleotide query) matches the $E$-value cutoff was $<1.0 \times 10^{-5}$.

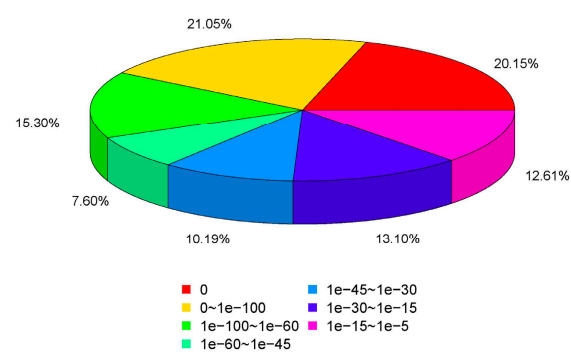

(A) $E$-values Distribution

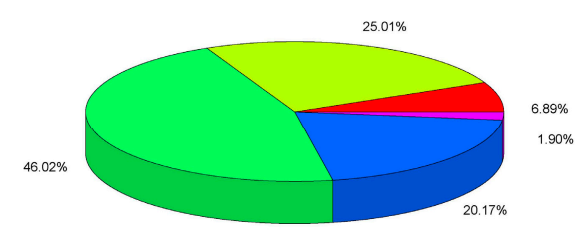

$=17 \% \sim 40 \%=80 \% \sim 95 \%$
$=40 \% \sim 60 \%=95 \% \sim 100 \%$
$=60 \% \sim 80 \%$

(B) Similarity Statistic

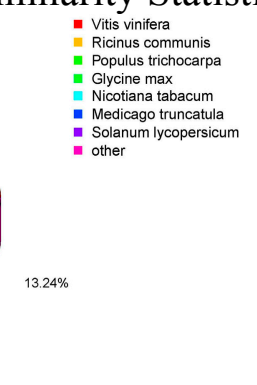

(C) Species Statistic

Figure 4. COG (Cluster of Orthologous Groups of proteins) classification of the $P$. fortunei all unigenes. 32,269 unigenes $(28.0 \%$ of the total) were annotated and grouped into 25 specific categories.

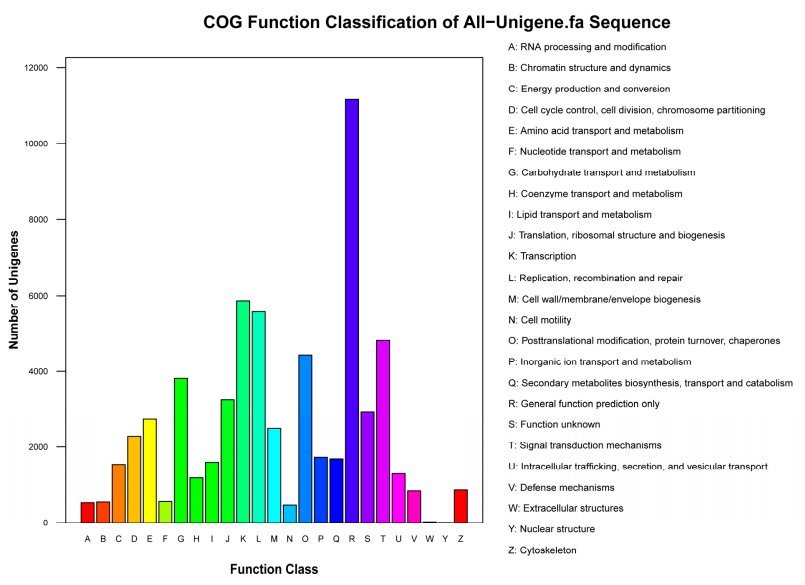


Figure 5. GO (Gene Ontology) classification of the $P$. fortunei all unigenes. 63,555 unigenes $(55.2 \%$ of total) were annotated and categorized into 57 function groups.

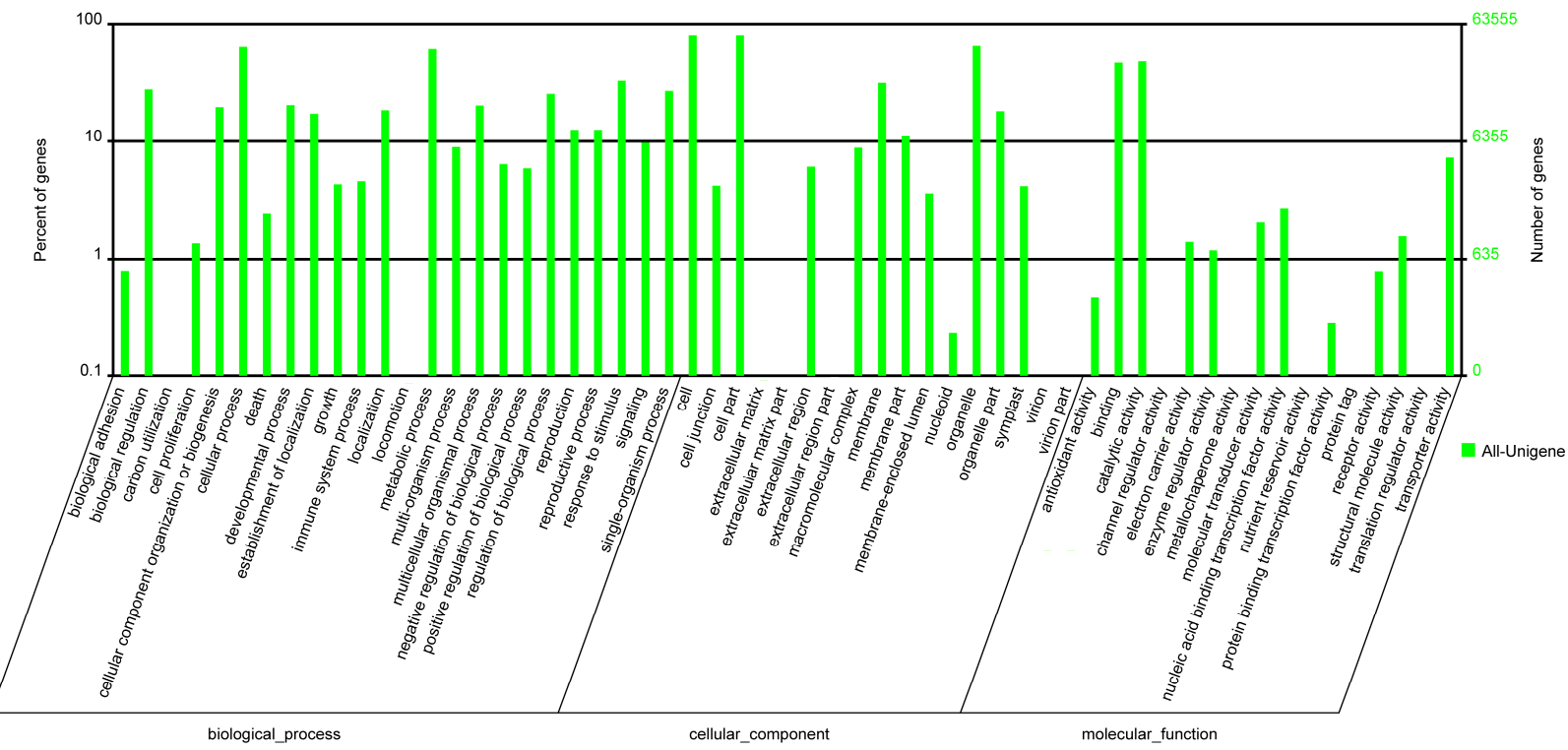

\subsection{Functional Classification of the Differentially Expressed Genes (DEGs)}

In total, 7612 DEGs (Differentially Expressed Genes) were identified in the PFI vs. PF transcriptome comparison (2643 were up-regulated and 3969 were down-regulated), and 2847 DEGs were identified in the PFI-60 vs. PFI transcriptome comparison (1970 were up-regulated and 877 were down-regulated) (Figure 6). DEGs that were up-regulated in the PFI vs. PF comparison and down-regulated in the PFI-60 vs. PFI comparison were retrieved (397 DEGs), and DEGs that were down-regulated in the PFI vs. PF comparison and up-regulated in the PFI-60 vs. PFI comparison were also retrieved (912 DEGs). These 1309 DEGs were taken to be the PaWB disease-related DEGs analyzed in this study (Table S2). Top DEGs and DEGs related to P. fortunei PaWB disease were listed in Table 3. Several genes, including brassinosteroid insensitive 1-associated receptor kinase1 (BAK1), mitogen-activated protein kinase kinasekinase 1 (MEKK1), and WRKY transcription factor 29 (WRKY29) that may be involved in the plant-pathogen interaction pathway. Other plant-pathogen-related genes such as $P B S 1$, which encodes a serine/threonine-protein kinase, and the transcription factor MYC2, were also identified among the DEGs. Adenylate isopentenyl transferase (IPT), two cytokinin trans-hydroxylases (CYP735A), and one cis-zeatin $O$-glucosyl transferase (CISZOG) that are involved in the biosynthesis of zeatin, abscisic acid receptor $(P Y L), \mathrm{ABA}$ responsive element binding factor $(A B F)$ and two lycopene cyclase $(\mathrm{CruA})$ that involved in carotenoid biosynthesis pathways were altered during phytoplasma infection. DEGs involved in light signal transduction, including genes that encode GIGANTEA $(G I)$, phytochrome-interacting factor 3 (PIF3), timing of CAB expression 1 (TOC1), circadian clock associated 1 $(C C A 1)$, and zinc finger protein CONSTANS $(C O)$ were also identified. 
Figure 6. Statistics of the $P$. fortunei DEG (Differetially Expressed Genes) numbers in each comparison. PF represents the healthy wild-type sample of $P$. fortunei, PFI represents the sample of phytoplasma infected PF. PFI-60 represents the sample of $60 \mathrm{mg} \cdot \mathrm{L}^{-1} \mathrm{MMS}$ treated PFI.

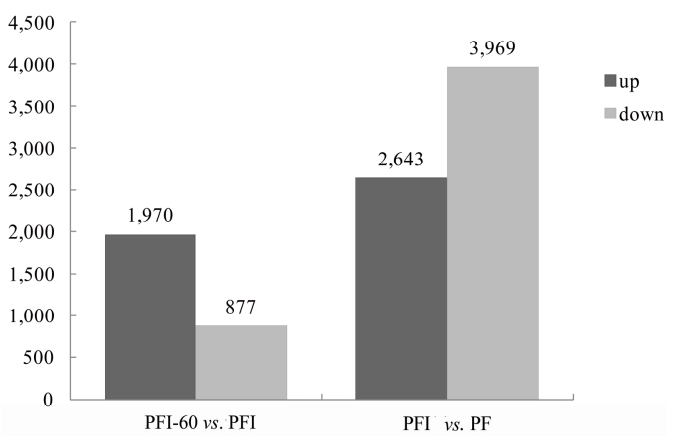

Table 3. Top up- and down-regulated differetially expressed genes (DEGs) and DEGs related to P. fortunei PaWB disease. DEGs up-regulated in the PFI vs. PF comparison and meanwhile down-regulated in the PFI-60 vs. PFI comparison were retrieved. So were the DEGs down-regulated in the PFI vs. PF comparison and meanwhile up-regulated in the PFI-60 vs. PFI comparison. PF represents the healthy wild-type sample of P. fortunei, PFI represents the sample of phytoplasma infected PF. PFI-60 represents the sample of $60 \mathrm{mg} \cdot \mathrm{L}^{-1} \mathrm{MMS}$ treated PFI.

\begin{tabular}{llll}
\hline Gene ID & Gene Length (bp) & Up/Down * & Potential Gene Function \\
\hline CL10743.Contig1 & 2028 & $\mathrm{Up}$ & Inositol-3-phosphate synthase \\
CL10743.Contig2 & 1969 & $\mathrm{Up}$ & Inositol-3-phosphate synthase \\
CL10796.Contig1 & 867 & $\mathrm{Up}$ & AT5G14920 \\
CL11352.Contig1 & 946 & $\mathrm{Up}$ & MLP-like protein 34 \\
CL3131.Contig1 & 2021 & $\mathrm{Up}$ & Uncharacterized protein LOC100260696 \\
CL6971.Contig3 & 1890 & $\mathrm{Up}$ & Cytochrome P450 \\
CL7523.Contig2 & 721 & $\mathrm{Up}$ & Gibberellin induced protein \\
Unigene10760 & 611 & $\mathrm{Up}$ & Plasma intrinsic protein 2.2 \\
Unigene12341 & 1172 & $\mathrm{Up}$ & Gibberellin induced protein \\
Unigene8595 & 323 & $\mathrm{Up}$ & PIP2 protein \\
Unigene8675 & 1282 & $\mathrm{Up}$ & UPF0301 protein Cpha266_0885-like isoform 1 \\
CL13170.Contig1 & 858 & $\mathrm{Up}$ & Transcription regulator \\
CL9733.Contig2 & 469 & $\mathrm{Up}$ & Glycine-rich protein \\
CL3432.Contig3 & 2432 & $\mathrm{Up}$ & Microtubule-associated protein TORTIFOLIA1 \\
CL13388.Contig1 & 370 & $\mathrm{Up}$ & Aquaporin PIP2 \\
Unigene44849 & 866 & $\mathrm{Up}$ & Cell wall-associated hydrolase \\
CL9691.Contig1 & 1215 & $\mathrm{Up}$ & Alpha-expansin 2 \\
Unigene43893 & 856 & $\mathrm{Up}$ & Hypothetical protein MTR_8g040260 \\
CL6181.Contig1 & 345 & $\mathrm{Up}$ & Major latex-like protein \\
CL3545.Contig3 & 2147 & $\mathrm{Up}$ & dof zinc finger protein DOF5.2 \\
CL2544.Contig6 & 850 & Down & Putative glycine-rich RNA binding protein \\
CL33.Contig2 & 2834 & Down & WD repeat-containing protein C2A9.03 \\
CL33.Contig3 & 5536 & Down & WD repeat-containing protein C2A9.03 \\
\hline & & &
\end{tabular}


Table 3. Cont.

\begin{tabular}{|c|c|c|c|}
\hline Gene ID & Gene Length (bp) & Up/Down * & Potential Gene Function \\
\hline CL33.Contig4 & 5626 & Down & WD-repeat protein \\
\hline CL33.Contig5 & 2815 & Down & WD repeat-containing protein $\mathrm{C} 2 \mathrm{~A} 9.03$ \\
\hline CL4119.Contig2 & 1113 & Down & Cytochrome P450 72A1 \\
\hline CL4119.Contig5 & 2185 & Down & CYP72A58 \\
\hline CL4739.Contig1 & 727 & Down & Phylloplanin \\
\hline CL4739.Contig2 & 610 & Down & Phylloplanin \\
\hline Unigene 10248 & 4052 & Down & WD repeat-containing protein $\mathrm{C} 2 \mathrm{~A} 9.03$ \\
\hline Unigene10249 & 1966 & Down & WD repeat-containing protein $\mathrm{C} 2 \mathrm{~A} 9.03$ \\
\hline Unigene22577 & 953 & Down & Unknown \\
\hline Unigene24555 & 3910 & Down & Unnamed protein product \\
\hline Unigene32594 & 743 & Down & Lipid transfer protein \\
\hline Unigene28962 & 1800 & Down & Glucose-6-phosphate/phosphate translocator 2 \\
\hline Unigene 30587 & 4360 & Down & GIGANTEA \\
\hline Unigene 30582 & 4161 & Down & GIGANTEA \\
\hline Unigene30581 & 4520 & Down & GIGANTEA \\
\hline CL4575.Contig2 & 2531 & Down & 1-Deoxy-D-xylulose-5-phosphate synthase \\
\hline Unigene23241 & 3053 & Down & WD repeat-containing protein $\mathrm{C} 2 \mathrm{~A} 9.03$ \\
\hline CL3202.Contig3 & 1081 & Up & WRKY protein WRKY29 \\
\hline Unigene 12008 & 1848 & Up & Histidine kinase CRE1 \\
\hline CL8962.Contig1 & 915 & Down & Histidine-containing phosphor transfer protein AHP \\
\hline CL12775.Contig1 & 533 & Up & Abscisic acid receptor PYL \\
\hline CL2960.Contig1 & 818 & Up & $\mathrm{ABA}$ responsive element binding factor $\mathrm{ABF}$ \\
\hline Unigene27866 & 1130 & Up & Adenylate isopentenyl transferase IPT \\
\hline CL4119.Contig2 & 1848 & Down & Cytokinin trans-hydroxylase CYP735A \\
\hline CL4119.Contig5 & 2185 & Down & Cytokinin trans-hydroxylase CYP735A \\
\hline CL2490.Contig6 & 1644 & Down & Serine/threonine-protein kinase PBS1 \\
\hline CL4203.Contig5 & 2362 & $\mathrm{Up}$ & Circadian clock associated 1 CCA1 \\
\hline Unigene30581 & 4520 & Down & GIGANTEA \\
\hline Unigene 10760 & 611 & Up & Plasma intrinsic protein 2 \\
\hline Unigene 14837 & 413 & Up & Plasma membrane $\mathrm{H}^{+}$-ATPase LilHA2 \\
\hline Unigene44439 & 940 & Up & Serine/threonine-protein kinase MEKK1 \\
\hline CL4362.Contig9 & 2426 & Down & Transcription factor MYC2 \\
\hline Unigene19249 & 1046 & Up & Zeatin $O$-glucosyl transferase CISZOG \\
\hline CL4203.Contig5 & 2326 & Up & Protein CCA1 \\
\hline CL4362.Contig9 & 2426 & Down & Transcription factor PIF3 \\
\hline CL13348.Contig14 & 3472 & Down & $\begin{array}{l}\text { Brassinosteroid insensitive 1-associated } \\
\text { receptor kinase } 1\end{array}$ \\
\hline CL4785.Contig7 & 2179 & Down & Serine/threonine protein kinase PBS1 \\
\hline Unigene27866 & 1130 & Up & Adenylate isopentenyl transferase IPT \\
\hline Unigene19249 & 1046 & Up & Cis-zeatin $O$-glucosyl transferase CISZOG \\
\hline CL2374.Contig8 & 2053 & Down & Lycopene cyclase CruA \\
\hline Unigene28043 & 937 & Up & Timing of CAB expression 1 TOC 1 \\
\hline CL12024.Contig1 & 877 & Up & Zinc finger protein CONSTANS \\
\hline
\end{tabular}

* Up or down regulation indicated the expression in the PFI vs. PF comparison. In the PFI-60 vs. PFI comparison, the tendencies were opposite. 
Tounderst and the functions of the selected DEGs and also find disease related genes, we use GO terms and KEGG pathways to predict their functions. The DEGs were annotated with 48 terms (Table 4) under the three main GO categories, biological process, cellular component, and molecular function (Figure S2), and 535 of the DEGs were assigned to 83 KEGG pathways (Table 5 and Table S3).

Table 4. GO function analysis results for the P. fortunei DEGs.

\begin{tabular}{|c|c|c|}
\hline Ontology & Class & Numbers of DEGs \\
\hline \multirow{24}{*}{ Biological process } & biological adhesion & 32 \\
\hline & biological regulation & 225 \\
\hline & cell proliferation & 9 \\
\hline & cellular component organization or biogenesis & 103 \\
\hline & cellular process & 482 \\
\hline & death & 44 \\
\hline & developmental process & 173 \\
\hline & establishment of localization & 119 \\
\hline & growth & 31 \\
\hline & immune system process & 43 \\
\hline & localization & 128 \\
\hline & metabolic process & 413 \\
\hline & multi-organism process & 98 \\
\hline & multicellular organismal process & 171 \\
\hline & negative regulation of biological process & 45 \\
\hline & pigmentation & 2 \\
\hline & positive regulation of biological process & 42 \\
\hline & regulation of biological process & 206 \\
\hline & reproduction & 126 \\
\hline & reproductive process & 126 \\
\hline & response to stimulus & 301 \\
\hline & rhythmic process & 17 \\
\hline & signaling & 58 \\
\hline & sulfur utilization & 2 \\
\hline \multirow{12}{*}{ Cellular component } & cell & 547 \\
\hline & cell junction & 21 \\
\hline & cell part & 547 \\
\hline & extracellular matrix & 1 \\
\hline & extracellular region & 78 \\
\hline & macromolecular complex & 55 \\
\hline & membrane & 230 \\
\hline & membrane part & 63 \\
\hline & membrane-enclosed lumen & 41 \\
\hline & organelle & 392 \\
\hline & organelle part & 102 \\
\hline & symplast & 21 \\
\hline
\end{tabular}


Table 4. Cont.

\begin{tabular}{ccc}
\hline Ontology & Class & Numbers of DEGs \\
\hline & antioxidant activity & 3 \\
binding & 341 \\
& catalytic activity & 307 \\
Molecular function & 22 \\
& electron carrier activity & 20 \\
& enzyme regulator activity & 13 \\
& molecular transducer activity & 25 \\
& nucleic acid binding transcription factor activity & 1 \\
& protein binding transcription factor activity & 3 \\
& nutrient reservoir activity & 3 \\
& receptor activity & 2 \\
& structural molecule activity & 53 \\
\hline
\end{tabular}

Table 5. TOP 10 KEGG pathway analysis results for the $P$. fortunei DEGs.

\begin{tabular}{llllll}
\hline$\#$ & Pathway & $\begin{array}{l}\text { DEGs with Pathway } \\
\text { Annotation }\end{array}$ & $\boldsymbol{p}$-Value & $\boldsymbol{Q}$ Value & $\begin{array}{l}\text { Pathway } \\
\text { ID }\end{array}$ \\
\hline 1 & Stilbenoid, diarylheptanoid & $30(5.61 \%)$ & $2.32 \times 10^{-18}$ & $1.92 \times 10^{-16}$ & ko00945 \\
& and gingerol biosynthesis & & & & \\
2 & Limonene and pinene degradation & $22(4.11 \%)$ & $2.20 \times 10^{-13}$ & $9.13 \times 10^{-12}$ & ko00903 \\
3 & Circadian rhythm-plant & $30(5.61 \%)$ & $3.34 \times 10^{-12}$ & $9.23 \times 10^{-11}$ & ko04712 \\
4 & RNA degradation & $35(6.54 \%)$ & $3.30 \times 10^{-11}$ & $6.85 \times 10^{-10}$ & ko03018 \\
5 & Carotenoid biosynthesis & $21(3.93 \%)$ & $8.94 \times 10^{-10}$ & $1.48 \times 10^{-8}$ & ko00906 \\
6 & Biosynthesis of secondary metabolites & $92(17.2 \%)$ & $3.82 \times 10^{-7}$ & $5.29 \times 10^{-6}$ & ko01110 \\
7 & Tryptophan metabolism & $11(2.06 \%)$ & $2.70 \times 10^{-6}$ & $3.21 \times 10^{-5}$ & ko00380 \\
8 & Porphyrin and chlorophyll metabolism & $15(2.8 \%)$ & $6.78 \times 10^{-6}$ & $6.85 \times 10^{-5}$ & ko00860 \\
9 & Monoterpenoid biosynthesis & $7(1.31 \%)$ & $7.43 \times 10^{-6}$ & $6.85 \times 10^{-5}$ & ko00902 \\
10 & Isoflavonoid biosynthesis & $6(1.12 \%)$ & $3.32 \times 10^{-5}$ & $2.76 \times 10^{-4}$ & ko00943 \\
\hline
\end{tabular}

\subsection{Confirmation of the RNA-Seq Expression Data by $q R T-P C R$}

To confirm the results of the transcriptome sequencing analysis, qRT-PCR was performed on 12 selected candidate DEGs. The primers used for the qRT-PCR analysis, potential gene functions, and amplicon sizes are shown in Table S4. cDNA fragments from the samples used for the transcriptome sequencing were used as templates. The expression patterns revealed by qRT-PCR (relative expressed level) of the allunigenes were consistent with their expression predicted by analysis of the DEG sequences (Figure 7). This result validated the gene expression variations obtained from our analysis of the unigene sequences. 
Figure 7. qRT-PCR analysis of candidate $P$. fortunei PaWB resistant genes. (The following genes were validated: WRKY transcription factor 29 (WRKY29), WRKY protein; CRE1, histidine kinase; $A H P$, histidine-containing phosphor transfer protein; $P Y L$, abscisic acid receptor; $A B F, \mathrm{ABA}$ responsive element binding factor; IPT, adenylate isopentenyl transferase; $C Y P 735 A$, cytokinin trans-hydroxylase; $P B S 1$, serine/threonine-protein kinase; CCA1, circadian clock associated 1; GI, GIGANTEA; Unigene10760, plasma intrinsic protein 2; Unigene 14837, plasma membrane $\mathrm{H}^{+}$-ATPase LilHA2. 18S rRNA was used as the internal reference gene. Standard error of the mean for three technical replicates is represented by the error bars. PF represents the healthy wild-type sample of $P$. fortunei. PFI represents the sample of phytoplasma infected PF. PTFI-60 represents the sample of $60 \mathrm{mg} \cdot \mathrm{L}^{-1} \mathrm{MMS}$ treated PFI).

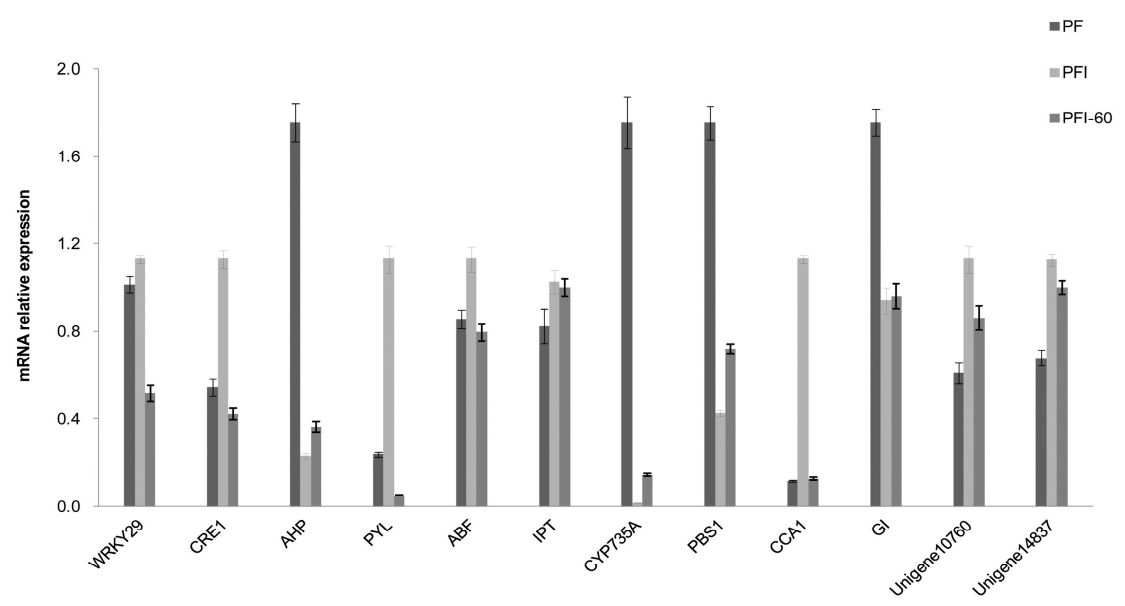

\section{Discussion}

The molecular mechanisms involved in the interaction between the PaWB phytoplasma and P. fortunei are largely unknown. Using RNA-Seq technology, we identified Paulownia genes and pathways whose expression profiles were altered among healthy, infected, and MMS-treated P. fortunei samples. Some of the differentially expressed sequences showed no similarity with sequences in the public databases, indicating that they may have novel important functions in the phytoplasma infection process and that they may be specific to $P$. fortunei.

Previously, we proposed a hypothesis about the plant-phytoplasma compatible interactions of $P$. tomentosa $\times$ P. fortunei [13]. Here, we retrieved the DEGs related to PaWB disease in $P$. fortunei and found that similar mechanisms may be involved. Based on the annotations of the final PaWB disease-related DEGs, we classified the response of $P$. fortunei to phytoplasma infection at the cellular and molecular levels. At the cellular level, the responses included modulation of the membrane system, modification of the cell wall, and alternation of the cell cycle and division. At the molecular level, the responses included transient alterations of the membrane ionic permeability such as $\mathrm{H}^{+}, \mathrm{Ca}^{2+}$, and $\mathrm{K}^{+}$. This response might be expected to initiate the synthesis and release of secondary messengers, including reactive oxygen species (ROS) (e.g., $\mathrm{H}_{2} \mathrm{O}_{2}$ ) that play significant roles in plant-pathogen reaction systems [15]. Elevated concentrations of ROS are noxious to plants, especially in some areas of the cells. Thus, antioxidants, such as cytochrome P450, are a necessary part of the defense response. High levels of the second messengers can cause a protein phosphorylation cascade reaction as has been reported 
in a similar study by [16]. Further, transcription factor (TF) expression might be activated, which would then initiated the expression of the related stress-induced genes, such as the enzymes involved in detoxin processes, the transporters of ions, the regulators of other TFs, protein kinases, and phosphatases. These data, to some extent, supported our previous study [13] and provided insights into the molecular mechanism of PaWB disease in $P$. fortunei.

In addition to the mechanisms described above, three different groups of DEGs associated with the response of $P$. fortunei to PaWB disease were identified.

\subsection{DEGs Related to Plant-Pathogen Interaction}

It has been reported that the inducible plant defense response to pathogens is multilayered and at least two stages are involved [17,18]. In the first stage, plant pattern recognition receptors trigger the recognition of pathogen-associated molecular patterns (PAMPs) [19], resulting in PAMP-triggered immunity. The second stage is initiated by the recognition of pathogen virulence proteins (effectors) or their activities by plant disease resistance (R) genes and the consequence is effector-triggered immunity [20]. PAMPs include the intracellular translation elongation factor Tu (EF-Tu). A conserved domain in EF-Tu, which can trigger PAMP-triggered immunity, has been recognized in Brassicaceae [21,22]. Phytoplasmas harbor genes that encode EF-Tu, and the products may induce PAMP-triggered immunity. In our study, we identified several genes, including BAK1, MEKK1, and WRKY29 that may be involved in the plant-pathogen interaction pathway. The pathway is shown in Figure 8. WRKY29 can induce the expression of defense-related genes, which include WRKY29 itself. Other plant-pathogen-related genes were also identified among the DEGs, such as $P B S 1$, which encodes a serine/threonine-protein kinase, and the transcription factor MYC2.

Figure 8. Elongation factor $\mathrm{Tu}(\mathrm{EF}-\mathrm{Tu})$ response pathway in P. fortunei. Genes in boxes are differently expressed. Red boxes indicate up-regulated or induced genes in the infected seedlings. Green boxes indicate down-regulated ones.

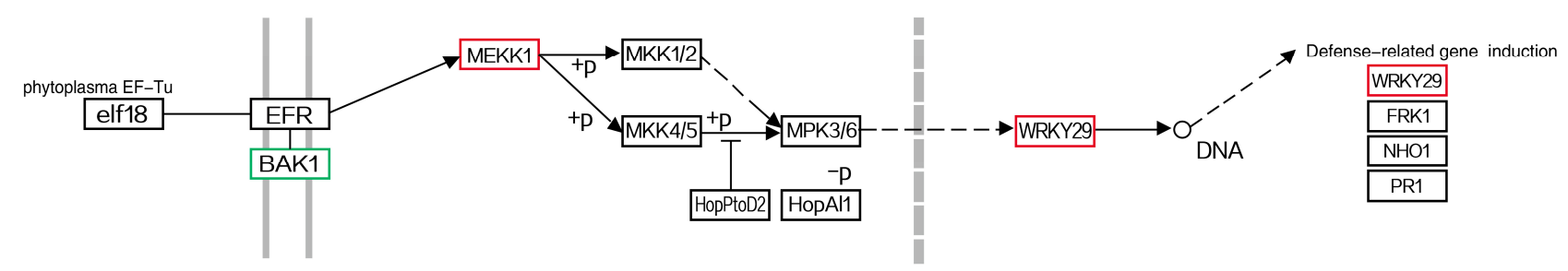

\subsection{DEGs Related to Hormone Biosynthesis}

During phytoplasma infection, plant hormones have been reported to be involved in symptom formation, such as witches' broom, smaller leaves, and short internodes [23,24]. Previous studies have found that elevated cytokinin (CK) levels can induce such symptoms. CKs can be synthesized through two major pathways, cis-zeatin and trans-zeatin, both of which need the presence of zeatin. Our analysis revealed several genes, including one IPT, two CYP735A, and one CISZOG that are involved in the biosynthesis of zeatin, which were differentially expressed in the process of phytoplasma infection (Table S1). It has been reported that in trans-zeatin biosynthesis, IPT is the first and key enzyme in the pathway [25-27]. Up-regulation of IPT in P. fortunei would elevate the concentration of CKs while 
down-regulation of $C Y P 735 A$ would probably affect the conversion of isopentenyladenine (iP)-nucleotide to $\mathrm{tZ}$ nucleotide. Thus, most CKs in P. fortunei may exist in the iP-type form. Furthermore, CRE1 and AHP that participate in the metabolism of $\mathrm{CK}$ were found to be differentially expressed. The overall effect of the genes described above would be to trigger cell division and shoot initiation.

Auxins have been reported to have anti-phytoplasma activity [28,29]. In our study, the genes related to auxin were found to be slightly down-regulated. As a result, the ratio of CK to auxin would be elevated, which could cause apical dominance and the formation of lateral buds (a symptom of witches' broom), as reported previously [30,31].

The stress-related hormone abscisic acid (ABA) has been shown to inhibit shoot growth and plant stem elongation [32,33]. We found among the DEGs associated with the carotenoid biosynthesis pathways that the expressions of $P Y L, A B F$ and two $C r u A$ were altered. These genes encode carotenoid precursors for ABA biosynthesis [34], which may induce stomatal closure and seed dormancy. Therefore, the formation of shorter internodes and smaller leaves in PaWB-infected plants may be associated with an increase in ABA.

In summary, we identified several genes related to $\mathrm{CK}$ and $\mathrm{ABA}$ biosynthesis that were differentially expressed after phytoplasma infection. Typical syndromes of phytoplasma infection seem to be associated with these genes. Therefore, $\mathrm{CK}$ and ABA may be important functional hormones in the response of Paulownia to witches' broom.

\subsection{DEGs Related to Circadian Rhythms}

Light provides a variety of signals for growth and development in higher plants. In the present study, several DEGs involved in light signal transduction were identified. The expressions of these genes were altered in the PFI samples compared with the PF samples. After MMS treatment, the alternations in expression returned to the expressions observed in the healthy (PF) samples (Figure 9).

Plants have evolved subtle photoreceptors system, such as phytochromes, cryptochromes, and phototropins, that regulate various light responses (e.g., blue light, far red light, or red light) through their interaction with different signaling proteins, among which the basic helix-loop-helix protein PIF3 is the most well-characterized [35]. This light-dependent regulator can induce light-regulated genes by binding to the phytochromes PHYA and PHYB. It has been proposed that PIF3 may act not only as a positive TF for chloroplast development, but also as a negative regulator for hypocotyl elongation [36]. A study on Arabidopsis thaliana seedlings reported that under diurnal light/dark conditions, PIF3 could target growth-related genes, and thus could also be considered a growth promoter [37]. In the present study, a gene that coded PIF3 was found to be down-regulated in PFI vs. PF and up-regulated in PFI-60 vs. PFI, which suggested that decreased light reception in the phytoplasma-infected Paulownia may contribute to the abnormal development of certain organs and decrease their sizes.

Changes in the gene expression patterns may alter the circadian rhythm and allow the plant to sense environmental light changes during the day/night cycle. Previous studies have showed that genes encoding TOC1, MYB, CCA1, and $L H Y$ work together as an autoregulatory loop, which could connect morning- and evening-phase circuits [38]. For example, it has been reported that when the expressions of $L H Y$ and $C C A 1$ rose in the morning the expression of the evening-expressed TOCl fell and, conversely, when the expressions of $L H Y$ and CCA1 fall, the expression of TOC1 rises [39]. In the present study, 
two genes that encode $T O C 1$ and $L H Y / C C A 1$, respectively, were altered in both the PFI $v s$. PF and PFI-60 $v s$. PFI comparisons and were also predicted to be involved in the circadian rhythm pathway in the KEGG analysis. Several metabolic developmental processes in plants have been reported to show a circadian rhythm in leaf movement and in stomata opening, and this has been correlated with the $\mathrm{CO}_{2}$ assimilation rate [40]. The DEGs related to circadian rhythm in the PFI sample may decrease the photosynthesis rate in the PFI and PFI-60 samples; thus, changing the growth rate of the plants.

Figure 9. Circadian rhythm pathway in P. fortunei. Genes in boxes are differently expressed. Red boxes indicate up-regulated or induced genes in the infected seedlings. Green boxes indicate down-regulated ones.

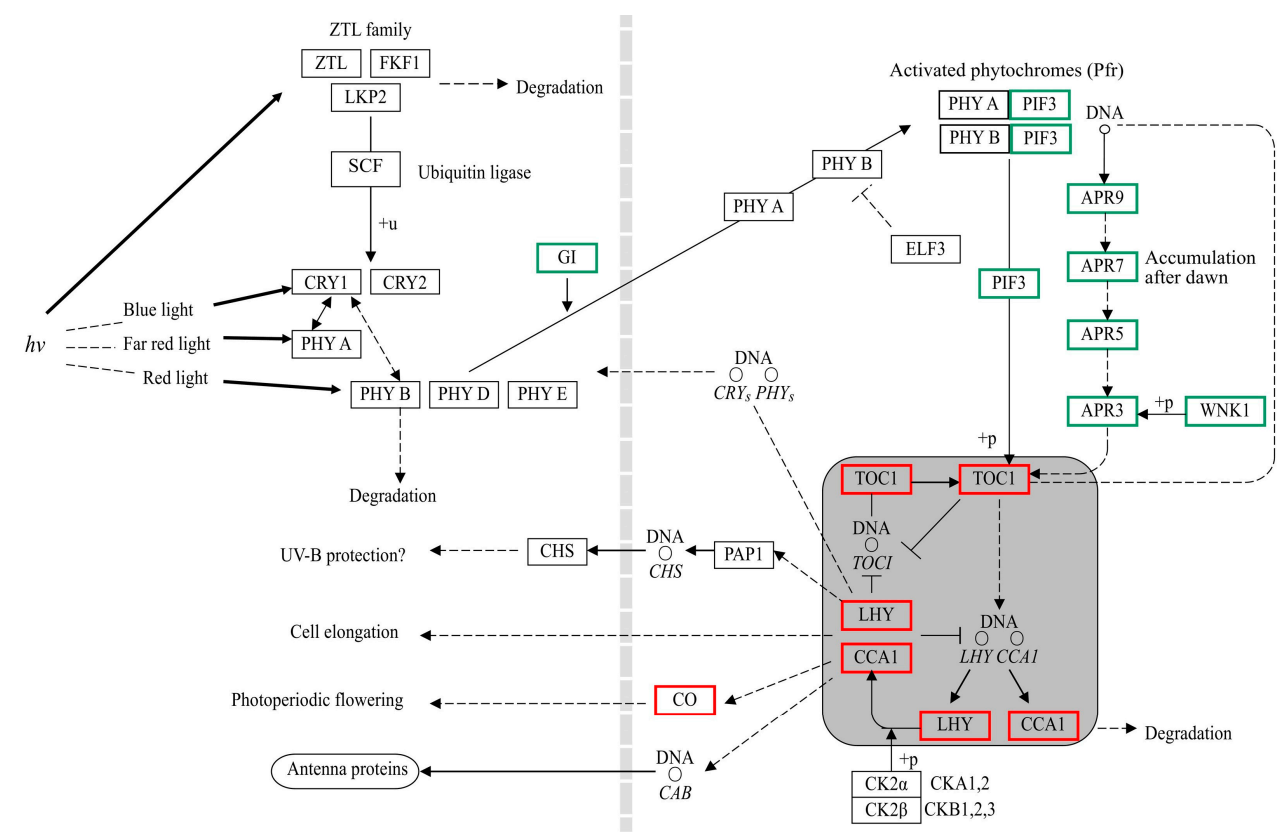

\section{Experimental Section}

\subsection{Preparation of Plant Material}

All of the biological material used in this study was obtained from the Institute of Paulownia, Henan Agricultural University, Zhengzhou, Henan Province, China. Tissue culture seedlings of P. fortunei, PF (healthy; three individuals) and PFI (PaWB-infected; six individuals) were cultured for 30 days before being clipped from the roots. The PFIs were transferred into $100-\mathrm{mL}$ triangular flasks containing $1 / 2 \mathrm{MS}$ culture medium [8] containing $25 \mathrm{mg} \cdot \mathrm{L}^{-1}$ sucrose and $8 \mathrm{mg} \cdot \mathrm{L}^{-1}$ agar (Sangon, Shanghai, China) with 0 or $60 \mathrm{mg} \cdot \mathrm{L}^{-1} \mathrm{MMS}$. The PFs were transferred into $100-\mathrm{mL}$ triangular flasks containing $1 / 2 \mathrm{MS}$ medium without MMS as the control. At least three parallel samples were prepared for each condition. All the samples were first cultured at $20{ }^{\circ} \mathrm{C}$ in the dark for 5 days. After that, they were cultured under $25 \pm 2{ }^{\circ} \mathrm{C}$ and $130 \mu \mathrm{mol} \cdot \mathrm{m}^{-2} \cdot \mathrm{s}^{-1}$ intensity light for $16 \mathrm{~h}$ every day.

\subsection{Construction of cDNA Libraries of P. fortunei}

For each condition, approximately $8 \mathrm{mg}$ of leaves were homogenized in liquid nitrogen with a pestle. Total RNA was extracted from the cells using TRIzol reagent (Invitrogen, Carlsbad, CA, USA), followed 
by RNA purification using the RNeasyMiniElute Cleanup Kit (Qiagen, Dusseldorf, Germany) according to the manufacturer's protocol. With a NanoVue UV-Vis spectrophotometer (GE Healthcare Bio-Science, Uppsala, Sweden), RNA was quantified by measuring absorbance at $260 \mathrm{~nm}$. Absorbance ratios of $\mathrm{OD}_{260 / 280}$ and OD260/230 were used to assess the purity of all the RNA samples. The integrity of the RNA was checked by $1 \%$ agarose gel electrophoresis. After total RNA extraction and DNase I treatment, magnetic beads with Oligo (dT) were used to isolate the mRNA. The mRNA was mixed with fragmentation buffer to obtain short fragments. cDNA was synthesized using the mRNA fragments as templates. The short cDNA fragments were purified and resolved with EB buffer for end reparation and single nucleotide A (adenine) addition. Then, the short fragments were connected with adapters. Suitable fragments were used as the templates for PCR amplification. An Agilent 2100 Bioanalyzer (Agilent Technologies, Palo Alto, CA, USA) and ABI StepOnePlus Real-Time PCR System (ABI, New York, NY, USA) were used to quantify and assess the quality of the sample library. Finally, the library was sequenced using IlluminaHiSeq ${ }^{\mathrm{TM}} 2000$ (Illumina, San Diego, CA, USA).

\subsection{Bioinformatics Analysis}

High-throughput RNA-seq was performed by Illumina RNA sequencing in Beijing Genomics Institute-Shenzhen (BGI-Shenzhen, Shenzhen, China). Base calling was used to transform the image data output from the sequencing machine into sequence data. Two software programs were used in this process: a real-time analyzer and the off-line basecaller (OLB). The real-time analyzer software, which was installed on the HiSeq ${ }^{\mathrm{TM}} 2000$ sequencer, can analyze the results while sequencing, and convert the image files into bcl files after analysis. The bcl files were output to the mainframe computer that ran the OLB software (version 1.9.4) to convert the bcl files into sequence data files. The input and output parameters (setupBclToQseq.py-b path/to/BaseCalls-o path/to/output/directory) used were the ordinary ones and no special parameters were involved. After that, another parameter (--ignore-missing-stats) was added to ignore the situations of missed files. The sequence data that were obtained were the raw reads and these data were stored in fastq format. The raw reads included low quality reads that contain adapters, and unknown or low quality bases. These contaminants were filtered out using the filter_fq program to produce the clean reads. The clean reads have been deposited in the NCBI Short Read Archive (SRA) database under accession number SRP040429. The Trinity short reads assembly software [41] was used to carry out de novo assembly of the clean reads. The assembled sequences were called unigenes. When multiple samples from the same species are sequenced, the unigenes from each assembly can be processed with sequence clustering software to remove sequence splicing and redundancy to acquire non-redundant unigenes that are as long as possible. Clustering of our unigene sequences generated clusters that contained several unigenes that shared more than $70 \%$ sequence similarity, these clusters were named using the prefix $\mathrm{CL}$, and the remaining unigenes that did not form clusters were the singletons, which were named using the prefix Unigene.

BLASTX alignments $\left(E\right.$-value $<1.0 \times 10^{-5}$ ) were performed between the assembled unigenes and sequences in the following protein databases: NCBI's non-redundant protein database (NR), Swiss-Prot, Kyoto Encyclopedia of Genes and Genomes (KEGG), and clusters of orthologous groups (COG). The sequence directions were decided based on the best alignment results over all these databases. When different databases generated conflicting results, the sequence direction was assigned based 
on a priority order of NR, Swiss-Prot, KEGG, and COG. When a unigene sequence could not be aligned to any of the sequences in the four databases, ESTS can [42] was used to decide its direction.

\subsection{Database Searches}

The CL and Unigene sequences were first aligned to the protein databases NR, Swiss-Prot, KEGG, and COG $\left(E\right.$-value cutoff was $\left.<1.0 \times 10^{-5}\right)$ using BLASTX, and to NCBI's nucleotide NT database $\left(E\right.$-value cutoff $<1.0 \times 10^{-5}$ ) using BLASTN. The protein sequences that shared the highest sequence similarity with the aligned unigenes were retrieved along with their Gene Ontology (GO) functional annotations. The KEGG database provided pathway annotations for the matched unigenes. The unigenes were also aligned to COG to help predict and classify the possible unigene functions.

\subsection{Unigene Gene Ontology (GO) Classification}

We used the Blast2GO program [43] to retrieve the GO annotations from the NR database for each of the matched unigenes. To understand the distribution of the functions of the genes in P. fortunei at the macro level, we used the WEGO software [44] to perform a GO functional classification for each unigene annotation.

\subsection{Protein Coding Sequence (CDS) Prediction}

The unigenes that matched sequences in the NR and Swiss-Prot databases were also aligned to KEGG and COG. Protein sequences with the highest ranks in the BLAST alignments were retrieved and used to decide the CDS of each unigene. The predicted CDSs were translated into amino sequences using the standard codon table to obtain both the nucleotide sequence (5'-3') and amino sequence for each unigene CDS. ESTS can [42] was used to predict the nucleotide sequence (5'-3') direction and the amino sequence for the unigenes that could not be aligned to any of the four databases.

\subsection{Unigene Expression Analysis}

We used the FPKM (fragments per kilobase of transcript per million mapped reads) method [45] to normalize read counts and determine the values of the expressed genes of the Paulownia transcriptomes, PFI vs. PF and PFI-60 vs. PFI. We have developed a rigorous algorithm based on Audic et al. [46] to identify DEGs between two samples. The null hypothesis and alternative hypothesis to identify DEGs were defined as: $H_{0}$, a gene with the same expression level in two samples; and $H_{1}$, a gene with different expression levels in two samples.

If $x$ is the number of fragments that can map uniquely to gene A, then for each transcript representing a small fraction of the library, $p(x)$ will closely follow the Poisson distribution [46] as:

$$
p(x)=\frac{e^{-\lambda} \lambda^{x}}{x !}
$$

where $\lambda$ is the real transcripts of the gene.

If the total number of fragments in sample 1 is $N 1$, and total number of fragments in sample 2 is $N 2$, and if gene A has $x$ fragments in sample 1 and $y$ fragments in sample 2, then the probability of gene A being expressed equally in the two samples can be calculated using the following formula [45]: 


$$
2 \sum_{i=0}^{\mathrm{y}} \mathrm{p}(i \mid \mathrm{x})\left(\text { while } \sum_{i=0}^{\mathrm{y}} \mathrm{p}(i \mid \mathrm{x}) \leq 0.5\right)
$$

or

$$
2\left(1-\sum_{i=0}^{\mathrm{y}} \mathrm{p}(i \mid \mathrm{x})\right)\left(\text { while } \sum_{i=0}^{\mathrm{y}} \mathrm{p}(i \mid \mathrm{x}) \geq 0.5\right)
$$

where

$$
p(i \mid x)=\left(\frac{\mathrm{N}_{2}}{\mathrm{~N}_{1}}\right)^{i} \frac{(x+i) !}{x ! i !\left(1+\left(\frac{\mathrm{N}_{2}}{\mathrm{~N}_{1}}\right)^{(\mathrm{x}+i+1)}\right.}
$$

After performing thousands of hypothesis tests, we found that a significant $p$-value for an individual test was not enough to guarantee a low false discovery rate (FDR). Therefore, we used a multiple testing correction method for each individual hypothesis testing to guarantee the FDR was low overall.

FDR control is a statistical method used in multiple hypothesis testing to correct for $p$-value. In practical terms, the FDR is the expected FDR; for example, if 1000 observations were predicted experimentally to be different and the maximum FDR for these observations was 0.1 , then 100 of these observations would be expected to be a false discovery, See [47] for details of this method. We then used the FDR along with the ratio of FPKMs for the two samples. The smaller the FDR is and the larger the ratio is, the larger the difference is in the expression levels between the two samples. A threshold FDR $\leq 1.0 \times 10^{-3}$ and an absolute value of $\log 2$ Ratio $>2$ were used to judge the significance of differences in gene expression.

\subsection{GO Functional Enrichment Analysis of the DEGs}

All the DEGs were first mapped to terms in the GO database and the numbers of genes annotated with each GO term were calculated to obtain a gene list and the numbers of genes for every assigned GO term. To identify significantly enriched GO terms among the DEGs, a hypergeometric test was used. The formula used to calculate the $p$-value in this hypothesis test was:

$$
P=1-\sum_{i=0}^{m-1} \frac{\left(\begin{array}{c}
M \\
i
\end{array}\right)\left(\begin{array}{c}
N-M \\
n-i
\end{array}\right)}{\left(\begin{array}{l}
N \\
n
\end{array}\right)}
$$

where $N$ is the number of all the genes with GO annotations, $n$ is the number of DEGs in $N$, $M$ is the number of all genes that are annotated to a certain GO term, and $m$ is the number of DEGs in $M$. The $p$-value calculated after a Bonferroni correction of $\leq 0.05$ was used as the threshold. GO terms that fulfilled this condition were defined as significantly enriched in the DEGs against the whole transcriptome background. The GO functional enrichment analysis was used to recognize the main biological functions of the DEGs and to integrate the clustering analysis of the expression patterns to easily obtain the expression patterns of the DEGs annotated with GO terms. 


\subsection{Kyoto Encyclopedia of Genes and Genomes (KEGG) Pathway Analysis of the DEGs}

Pathway enrichment analysis was used to retrieve significantly enriched pathways in the DEGs against the whole transcriptome background. A similar formula to the one used to calculate the $p$-value in the GO analysis was used. A $q$-value was defined as the FDR analog of the $p$-value. After multiple testing and correction, we choose a $q$-value of $\leq 0.05$ to identify the pathways that were significantly enriched in the DEGs. The parameters of all the software used in this study were shown in Table S5.

\subsection{Quantitative Real-time PCR ( $q R T-P C R)$ Analysis}

The RNA samples from the leaves of the PF, PFI, and PFI-60 samples were extracted with Trizol (Sangon, Shanghai, China). The RNA was then precipitated with isopropanol. Purified and concentrated RNA was denatured and first-strand cDNAs for all the samples were synthesized using a PrimeScript RT reagent Kit (Takara, Dalian, China). Potential genes related to PaWB disease resistance were chosen. Primers were designed with Beacon Designer, version 7.7 (Premier Biosoft International, Ltd., Palo Alto, CA, USA). The cDNAs were then amplified in a Bio-Rad CFX96TM Real-Time System (Bio-Rad, Hercules, CA, USA) with SYBR Premix Ex Taq TM II (Takara, Dalian, China). The following PCR parameters were used: $50{ }^{\circ} \mathrm{C}$ for $2 \mathrm{~min}, 95^{\circ} \mathrm{C}$ for $30 \mathrm{~s}$, followed by 40 cycles of $94{ }^{\circ} \mathrm{C}$ for $15 \mathrm{~s}$ and $60{ }^{\circ} \mathrm{C}$ for $1 \mathrm{~min}$. Three replicates were analyzed for each gene. The average threshold cycle $\left(C_{\mathrm{t}}\right)$ was normalized and the relative expression changes were calculated using the $2^{-\Delta \Delta C t}$ method. The $18 \mathrm{~S}$ rRNA of Paulownia was chosen as an internal reference gene for normalization.

\section{Conclusions}

In summary, this investigation provides the first transcriptomic analysis of phytoplasma-infected $P$. fortunei and the transcriptome data have enriched our knowledge of $P$. fortunei. Further, the analysis of the DEGs in healthy, phytoplasma-infected, and MMs-treated phytoplasma-infected Paulownia seedlings revealed a number of candidate genes and inherent pathways related to the PaWB disease symptoms, which has shed some light on the molecular mechanisms associated with this biological process. Although a large number of novel disease-related DEGs remain unannotated, the current study has established a substantial platform for in-deep studies into the mechanisms in Paulownia that are affected by the biotic stress caused by phytoplasma infection. There are common mechanisms in the process of photoplasma infection between different Paulownia species. However, some different mechanisms and a lot of DEGs from other Paulownia species have also been found. Further, this study provides new insights into the response and resistance of $P$. fortunei against PaWB disease.

\section{Supplementary Materials}

Supplementary materials can be found at http://www.mdpi.com/1422-0067/15/12/23141/s1.

\section{Acknowledgments}

We would like to give our thanks to Beijing Genomics Institute-Shenzhen (BGI-Shenzhen) for helping us with the throughput RNA-seq analysis. This work was supported by the Joint Funds of the National 
Natural Science Foundation of China (NSFC) (U1204309), the Fund of the Transformation Project of the National Agricultural Scientific and Technological Achievement of China (2012GB2D000271), the Central Financial Forestry Science Promotion Project (GTH [2012]01), the Fund of the Science Key Program of Department of Henan Education (12A220003 and 14A220001), the Fund of the Technology Innovation Team Project of Zhengzhou (121PCXTD515) and the Fund of Zhongyuan Scholarship Foundation of Henan Province (122101110700).

\section{Author Contributions}

Guoqiang Fan conceived and designed the experiments; Minjie Deng performed the experiments; Zhenli Zhao analyzed the data; Suyan Niu and Enkai Xu contributed reagents/materials/analysis tools; Yanpeng Dong wrote the paper.

\section{Conflicts of Interest}

The authors declare no conflict of interest.

\section{References}

1. Nakamura, H.; Yoshikawa, N.; Takahashi, T.; Sahashi, N.; Kubono, T.; Shoji, T. Evaluation of primer pairs for the reliable diagnosis of Paulownia witches'-broom disease using a polymerase chain reaction.Plant Dis. 1996, 80, 302-305.

2. Hiruki, C. Paulownia witches'-broom disease important in East Asia. Int. Symp. Urban Tree Health 1997, 469, 63-68.

3. Namba, S. Molecular biological studies on phytoplasmas. J. Gen. Plant Pathol. 2002, 68, 257-259.

4. Bayliss, K.L.; Saqib, M.; Dell, B.; Jones, M.G.K.; Hardy, G.E.S.J. First record of "Candidatus Phytoplasma australiense" in Paulownia trees. Australas. Plant Path. 2005, 34, 123-124.

5. Doi, M.; Tetranaka, M.; Yora, K.; Asuyama, H. Mycoplasma or PLTgroup-like organisms found in the phloem elements of plants infected with mulberry dwarf, potato witches' broom, aster yellows or Paulownia witches' broom. Ann. Phytopathol. Soc. Jpn. 1967, 33, 259-266.

6. Group., T.I.P.; Firrao, G. “Candidatus Phytoplasma”, a taxon for the wall-less, nonhelical prokaryotes that colonize plant phloem and insects. Int. J. Syst. Evol. Microbiol. 2004, 54, 1243-1255.

7. Fan, G.; Zhang, S.; Zhai, X.; Liu, F.; Dong, Z. Effects of antibiotics on the Paulownia witches' broom phytoplasmas and pathogenic protein related to witches' broom symptom. Sci. Silv. Sin. 2007, 43, 138-142.

8. Zhai, X.; Cao, X.; Fan, G. Growth of Paulownia witche' broom seedlings treated with methyl methane sulphonate and SSR analysis. Sci. Silv. Sin. 2010, 46, 176-181.

9. Cao, X.; Fan, G.; Deng, M.; Zhao, Z.; Dong, Y. Identification of genes related to Paulownia witche' broom by AFLP and MSAP. Int. J. Mol. Sci. 2014, 15, 14669-14683.

10. Heinrich, M.; Botti, S.; Caprara, L.; Arthofer, W.; Strommer, S.; Hanzer, V.; Katinger, H.; Bertaccini, A.; Machado, M.L.D.C. Improved detection methods for fruit tree phytoplasmas. Plant Mol. Biol. Rep. 2001, 19, 169-179. 
11. Baric, S.; Kerschbamer, C.; Dalla Via, J. TaqMan real-time PCR vs. four conventional PCR assays for detection of apple proliferation phytoplasma. Plant Mol. Biol. Rep. 2006, 24, 169-184.

12. Mou, H.Q.; Lu, J.; Zhu, S.F.; Lin, C.L.; Tian, G.Z.; Xu, X.; Zhao, W.J. Transcriptomic analysis of Paulownia infected by Paulownia witches'-broom Phytoplasma. PLoS One 2013, 8, e77217.

13. Liu, R.; Dong, Y.; Fan, G.; Zhao, Z.; Deng, M.; Cao, X.; Niu, S. Discovery of genes related to witches broom disease in Paulownia tomentosa $\times$ Paulownia fortunei by a de novo assembled transcriptome. PLoS One 2013, 8, e80238.

14. Lee, I.M.; Hammond, R.; Davis, R.; Gundersen, D. Universal amplification and analysis of pathogen 16S rDNA for classification and identification of mycoplasmalike organisms. Phytopathology 1993, 83, 834-842.

15. Durrant, W.E.; Rowland, O.; Piedras, P.; Hammond-Kosack, K.E.; Jones, J.D. cDNA-AFLP reveals a striking overlap in race-specific resistance and wound response gene expression profiles. Plant Cell Online 2000, 12, 963-977.

16. Xiong, L.; Schumaker, K.S.; Zhu, J.K. Cell signaling during cold, drought, and salt stress. Plant Cell 2002,14, S165-S183.

17. Jones, J.D.; Dangl, J.L. The plant immune system. Nature 2006, 444, 323-329.

18. Oshima, K.; Kakizawa, S.; Nishigawa, H.; Jung, H.Y.; Wei, W.; Suzuki, S.; Arashida, R.; Nakata, D.; Miyata, S.I.; Ugaki, M. Reductive evolution suggested from the complete genome sequence of a plant-pathogenic phytoplasma. Nat. Genet. 2004, 36, 27-29.

19. Schwessinger, B.; Zipfel, C. News from the frontline: Recent insights into PAMP-triggered immunity in plants. Curr. Opin. Plant Biol. 2008, 11, 389-395.

20. Dodds, P.N.; Rathjen, J.P. Plant immunity: Towards an integrated view of plant-pathogen interactions. Nat. Rev. Genet. 2010, 11, 539-548.

21. Zipfel, C.; Kunze, G.; Chinchilla, D.; Caniard, A.; Jones, J.D.; Boller, T.; Felix, G. Perception of the bacterial PAMP EF-Tu by the receptor EFR restricts Agrobacterium-mediated transformation. Cell 2006, 125, 749-760.

22. Kunze, G.; Zipfel, C.; Robatzek, S.; Niehaus, K.; Boller, T.; Felix, G. The $N$ terminus of bacterial elongation factor Tu elicits innate immunity in Arabidopsis plants. Plant Cell Online 2004, 16, 3496-3507.

23. Christensen, N.M.; Axelsen, K.B.; Nicolaisen, M.; Schulz, A. Phytoplasmas and their interactions with hosts. Trends Plant Sci. 2005, 10, 526-535.

24. Hoshi, A.; Oshima, K.; Kakizawa, S.; Ishii, Y.; Ozeki, J.; Hashimoto, M.; Komatsu, K.; Kagiwada, S.; Yamaji, Y.; Namba, S. A unique virulence factor for proliferation and dwarfism in plants identified from a phytopathogenic bacterium. Proc. Natl. Acad. Sci. USA 2009, 106, 6416-6421.

25. Miyawaki, K.; Tarkowski, P.; Matsumoto-Kitano, M.; Kato, T.; Sato, S.; Tarkowska, D.; Tabata, S.; Sandberg, G.; Kakimoto, T. Roles of Arabidopsis ATP/ADP isopentenyltransferases and tRNA isopentenyltransferases in cytokinin biosynthesis. Proc. Natl. Acad. Sci. USA 2006, 103, 16598-16603.

26. Kakimoto, T. Biosynthesis of cytokinins. J. Plant Res. 2003, 116, 233-239.

27. Kamada-Nobusada, T.; Sakakibara, H. Molecular basis for cytokinin biosynthesis. Phytochemistry 2009, 70, 444-449. 
28. Ćurković Perica, M. Auxin-treatment induces recovery of phytoplasma-infected periwinkle. J. Appl. Microbiol. 2008, 105, 1826-1834.

29. Weintraub, P.G.; Jones, P. Phytoplasmas: Genomes, Plant Hosts and Vectors; CABI: Wallingford, UK, 2010.

30. Medford, J.I.; Horgan, R.; El-Sawi, Z.; Klee, H.J. Alterations of endogenous cytokinins in transgenic plants using a chimeric isopentenyl transferase gene. Plant Cell Online 1989, 1, 403-413.

31. Cline, M.; Wesse, T.; Iwamura, H. Cytokinin/auxin control of apical dominance in Ipomoea nil. Plant Cell Physiol. 1997, 38, 659-667.

32. Arney, S.; Mitchell, D. The effect of abscisic acid on stem elongation and correlative inhibition. New Phytol. 1969, 68, 1001-1015.

33. Kaufman, P.B.; Jones, R.A. Regulation of growth in Avena (Oat) stem segments by gibberellic acid and abscisic acid. Physiol. Plant. 1974, 31, 39-43.

34. Nambara, E.; Marion-Poll, A. Abscisic acid biosynthesis and catabolism. Annu. Rev. Plant Biol. 2005, 56, 165-185.

35. Ni, M.; Tepperman, J.M.; Quail, P.H. PIF3, a phytochrome-interacting factor necessary for normal photoinduced signal transduction, is a novel basic helix-loop-helix protein. Cell 1998, 95, $657-667$.

36. Stephenson, P.G.; Fankhauser, C.; Terry, M.J. PIF3 is a repressor of chloroplast development. Proc. Natl. Acad. Sci. USA 2009, 106, 7654-7659.

37. Soy, J.; Leivar, P.; González-Schain, N.; Sentandreu, M.; Prat, S.; Quail, P.H.; Monte, E. Phytochrome-imposed oscillations in PIF3 protein abundance regulate hypocotyl growth under diurnal light/dark conditions in Arabidopsis. Plant J. 2012, 71, 390-401.

38. Levy, Y.Y.; Dean, C. Control of flowering time. Curr. Opin. Plant Biol. 1998, 1, 49-54.

39. Alabadí, D.; Oyama, T.; Yanovsky, M.J.; Harmon, F.G.; Mas, P.; Kay, S.A. Reciprocal regulation between TOC1 and LHY/CCA1 within the Arabidopsis circadian clock. Science 2001, 293, 880-883.

40. Wang, Z.; Tobin, E.M. Constitutive expression of the CIRCADIAN CLOCK ASSOCIATED 1 (CCA1) gene disrupts circadian rhythms and suppresses its own expression. Cell 1998, 93, 1207-1217.

41. Grabherr, M.G.; Haas, B.J.; Yassour, M.; Levin, J.Z.; Thompson, D.A.; Amit, I.; Adiconis, X.; Fan, L.; Raychowdhury, R.; Zeng, Q.; et al. Full-length transcriptome assembly from RNA-Seq data without a reference genome. Nat. Biotechnol. 2011, 29, 644-652.

42. Iseli, C.; Jongeneel, C.V.; Bucher, P. ESTScan: A program for detecting, evaluating, and reconstructing potential coding regions in EST sequences. Proc. Int. Conf. Intell. Syst. Mol. Biol. 1999, 99, 138-148.

43. Conesa, A.; Gotz, S.; Garcia-Gomez, J.M.; Terol, J.; Talon, M.; Robles, M. Blast2GO: A universal tool for annotation, visualization and analysis in functional genomics research. Bioinformatics 2005, 21, 3674-3676.

44. Ye, J.; Fang, L.; Zheng, H.; Zhang, Y.; Chen, J.; Zhang, Z.; Wang, J.; Li, S.; Li, R.; Bolund, L.; et al. WEGO: A web tool for plotting GO annotations. Nucleic Acids Res. 2006, 34, W293-W297. 
45. Mortazavi, A.; Williams, B.A.; McCue, K.; Schaeffer, L.; Wold, B. Mapping and quantifying mammalian transcriptomes by RNA-Seq. Nat. Methods 2008, 5, 621-628.

46. Audic, S.; Claverie, J.M. The significance of digital gene expression profiles. Genome Res. 1997, 7, 986-995.

47. Benjamini, Y.; Drai, D.; Elmer, G.; Kafkafi, N.; Golani, I. Controlling the false discovery rate in behavior genetics research. Behav. Brain Res. 2001, 125, 279-284.

(C) 2014 by the authors; licensee MDPI, Basel, Switzerland. This article is an open access article distributed under the terms and conditions of the Creative Commons Attribution license (http://creativecommons.org/licenses/by/4.0/). 